\title{
Generating multi-GeV electron bunches using single stage laser wakefield acceleration in a 3D nonlinear regime
}

\author{
W. Lu, M. Tzoufras, and C. Joshi \\ Department of Electrical Engineering, University of California, Los Angeles, California 90095, USA \\ F. S. Tsung \\ Department of Physics and Astronomy, University of California, Los Angeles, California 90095, USA \\ W. B. Mori \\ Department of Electrical Engineering, University of California, Los Angeles, California 90095, USA, \\ and Department of Physics and Astronomy, University of California, Los Angeles, California 90095, USA \\ J. Vieira, R. A. Fonseca, and L. O. Silva \\ GoLP/Centro de Fisica dos Plasmas, Instituto Superior Téchnico, 1049-001 Lisboa, Portugal
}

(Received 19 December 2006; published 5 June 2007)

\begin{abstract}
The extraordinary ability of space-charge waves in plasmas to accelerate charged particles at gradients that are orders of magnitude greater than in current accelerators has been well documented. We develop a phenomenological framework for laser wakefield acceleration (LWFA) in the 3D nonlinear regime, in which the plasma electrons are expelled by the radiation pressure of a short pulse laser, leading to nearly complete blowout. Our theory provides a recipe for designing a LWFA for given laser and plasma parameters and estimates the number and the energy of the accelerated electrons whether self-injected or externally injected. These formulas apply for self-guided as well as externally guided pulses (e.g. by plasma channels). We demonstrate our results by presenting a sample particle-in-cell (PIC) simulation of a $30 \mathrm{fs}, 200 \mathrm{TW}$ laser interacting with a $0.75 \mathrm{~cm}$ long plasma with density $1.5 \times 10^{18} \mathrm{~cm}^{-3}$ to produce an ultrashort (10 fs) monoenergetic bunch of self-injected electrons at $1.5 \mathrm{GeV}$ with $0.3 \mathrm{nC}$ of charge. For future higher-energy accelerator applications, we propose a parameter space, which is distinct from that described by Gordienko and Pukhov [Phys. Plasmas 12, 043109 (2005)] in that it involves lower plasma densities and wider spot sizes while keeping the intensity relatively constant. We find that this helps increase the output electron beam energy while keeping the efficiency high.
\end{abstract}

DOI: 10.1103/PhysRevSTAB.10.061301

PACS numbers: 52.38.Kd, 52.35.Mw, 52.65.Rr

\section{INTRODUCTION}

In plasma based acceleration, a laser or particle beam creates a plasma wave wakefield with a phase velocity close to the speed of light $[1,2]$. The acceleration gradients in these wakefields can easily exceed $10 \mathrm{GeV} / m$ which is nearly 3 orders of magnitude larger than that achieved in conventional rf technology. A particle injected in such a wave with sufficient initial energy can interact with the longitudinal component of the electric field for a long enough time, that its energy gain is significant.

An intense laser pulse or electron beam can expel the plasma electrons outward to create a bare ion column [35], which has ideal accelerating and focusing properties for electron beams [4,5]. A recent important development in this field has been the observations in simulations that these highly nonlinear 3D wakefield structures remain stable [6-9]. In the laser-driven cases, the simulations showed that self-injected quasimonoenergetic beams of electrons with energies on the order of $100 \mathrm{MeV}$ can be formed [6,7]. In experiments where parameters were chosen such that complete electron expulsion was expected, monoenergetic electron beams were observed
[10-15]. In simulations of these experiments it was found that the energetic electron generation mechanism involves an interplay of complex phenomena: namely, the evolution of the laser, the self-trapping of electrons, and their influence on the wake [16]. The experimental observation of these structures may soon be possible using new diagnostic techniques [17].

In this article, we describe how to extend the results from the recent experiments towards a more stable regime conducive to making an accelerator. We will show that by using near term lasers in the 0.1 to $3 \mathrm{PW}$ range it will be possible to generate $1-13 \mathrm{GeV}$ monoenergetic electron beams with $\mathrm{nC}$ of charge in a single stage without the need for external guiding. Using external guiding energies up to $120 \mathrm{GeV}$ can be achieved in a single stage for a $3 \mathrm{PW}$ laser. Unlike in the recent experiments, this regime is characterized by lasers whose intensity and spot size are matched and by relatively low plasma densities. The matched laser profile (both transverse and longitudinal) evolves little during the acceleration distance which greatly exceeds the Rayleigh length.

We are motivated to determine a path towards making a compact electron accelerator based on the laser wakefield 
acceleration (LWFA) concept for use in high-energy physics or as a light source. Efficiency and beam quality are key issues one should keep in mind when making such designs. As shown by Katsouleas et al. [18], in order to achieve sufficient beam loading efficiency (from wake to particles) and to reduce the beam emittance growth, the spot size of the wake (hence laser) should be less than a plasma skin depth. In addition, in order to most efficiently transfer the laser energy to the wake over the entire effective accelerating distance (pump depletion length matching dephasing length), the laser normalized vector potential (laser intensity) must be larger than unity. These two conditions are based on linear theory, however, taken together they imply that LWFA needs to be operated in a 3D nonlinear regime, in which the laser power exceeds the critical power for relativistic self-focusing [3].

It was shown by Sun et al. [3] that for intense and narrow lasers the transverse ponderomotive force can lead to complete electron blowout (cavitation) when the laser power slightly exceeds the critical power for relativistic selffocusing, $P_{c}$, and the laser is focused tightly. This analysis was done for long pulses (although it assumed the ions were fixed). Mora and Antonsen [19] showed in reduced particle-in-cell (PIC) simulations that cavitation could occur for laser power above $P_{c}$. When using ultrashort pulses, $c \tau \lesssim w_{0} \simeq 2 \sqrt{a_{0}} c / \omega_{p}$, where $a_{0}=e A_{\text {laser }} /\left(m c^{2}\right)$ for a linearly polarized laser and $w_{0}$ the laser spot size, the ions are indeed stationary, and a region nearly void of electrons (ion channel) is generated behind the laser pulse. This channel exerts an attractive Coulomb force on the blown-out electrons causing them to rush back toward the axis thereby exciting a wakefield. In 1991 Rosenzweig et al. [4] showed that wakes excited in this nonlinear blowout regime (they used a particle beam driver) had ideal focusing and accelerating properties. Very soon thereafter it was shown using 2D simulations that similar wakes could be made by lasers [5].

In 2002, Puhkov and Meyer-ter-vehn used 3D particlein-cell simulations of LWFA at very high laser intensities and plasma densities without external guiding and found that electrons were self-injected in the blowout regime and that they formed a quasimonoenergetic beam as they were accelerated [6]. In their simulations the blowout cavity formed a sphere leading them to subsequently call this the "bubble" regime for LWFA [20]. Recently, a theoretical analysis [21,22] showed that, for sufficiently intense and ultrashort lasers, $a_{0} \geqslant 4$, the resulting electron density structure will resemble a spherical cavity and it was shown that the laser spot size needed to be matched to the maximum blowout (bubble) radius in order to achieve the most ideal wakes. It is worth noting for $2 \lesssim a_{0} \lesssim 4$ electron blowout still occurs with the cavity slightly deviating from a spherical shape.

The recent experimental results are promising and can be useful for many applications [23], but they still rely on substantial evolution of the laser and the energy spread and emittances are not sufficient for high-energy physics applications. What paths are available for extending these experiments towards higher energies with more control using the bubble regime? The original simulation results on the bubble regime in Ref. [6] were based on a simulation of a $360 \mathrm{TW}$ laser propagating through a 0.01 critical density $\left(1.74 \times 10^{19} \mathrm{~cm}^{-3}\right.$ for $\left.\lambda=0.8 \mu \mathrm{m}\right)$ plasma. Do the key features of this simulation remain for different laser powers, pulse lengths, spot sizes, and plasma densities? Answering these questions is difficult because the inherent physics is highly nonlinear.

One approach was put forth by Gordienko and Puhkov (GP) [24]. They argued that for $a_{0} \gg 1$ the speed of all plasma electrons is very close to the speed of light and under this condition all quantities will scale with a single similarity parameter, $S \equiv \frac{n_{p}}{n_{c} a_{0}}$, to some power. The coefficients in front of the scalings are determined from simulations (or experiments). The resulting expressions are therefore only strictly valid so long as the laser's transverse and longitudinal profile, aspect ratio, etc. remain the same. Another approach presented here is to use a phenomenological description. We identify the important physics as wake excitation (amplitude and phase velocity), pump depletion (pulse evolution), dephasing (between particles and wake), and beam loading. We use these concepts to develop expressions for predicting the number of electrons, the electron energy, and overall efficiency.

For any useful accelerator, the accelerating structure's (wake) amplitude, wavelength, and phase velocity need to be reasonably stable during the entire acceleration process. We show that a stable wake can be excited in the blowout regime when the laser's spot size is roughly matched to the blowout radius. We also show that under these conditions expressions for the wake amplitude and phase velocity (dephasing length), the laser pump depletion length, and the number of accelerated electrons can then be obtained including their coefficients based on simple physics arguments and previously published ideas. Importantly, unlike when using a similarity theory, the coefficients derived from the phenomenological theory can be extended to propagation in plasma channels as well as to externally injected electron beams.

We will show that, while there is overlap in the ideas put forth here and by GP, there are also profound differences besides those just described. Their analysis can only be valid when $a_{0} \geq 2 \sqrt{n_{c} / n_{p}}$ while ours is for $a_{0} \gtrsim 2$. Their supporting simulations used $a_{0}=20-80$ (for each direction of a circularly polarized laser which is the same as 28-113 for a linearly polarized laser) and $n / n_{c}=$ $0.02-0.08$. We will show that in contrast to the work of GP, our expressions advocate using lower $a_{0}$ and lower densities. In fact, we will argue that in order to obtain controlled acceleration one should operate with an $a_{0}$ too low for the key assumption in the theory of GP to even 
apply. Despite these differences we borrow the term bubble from Ref. [20] to describe the near spherical ion channel behind the laser pulse.

To verify our results, we have carried out many computer experiments using the $3 \mathrm{D}$, particle-in-cell code OSIRIS [25], that explore a wide range of plasma densities, laser powers, and spot sizes (see later). We highlight one simulation that is very relevant to near term experiments. Details of the simulation parameters and setup are provided in the Appendix. In this simulation, a $30 \mathrm{fs}$ (FWHM) $0.8 \mu \mathrm{m}$ laser pulse containing $200 \mathrm{TW}$ of power is focused to a spot size $w_{0}=19.5 \mu \mathrm{m}$ at the entrance of a $1.5 \times$ $10^{18} \mathrm{~cm}^{-3}$ density plasma to give a normalized vector potential of $a_{0}=4$. The laser is circularly polarized (with normalized vector potential $4 / \sqrt{2}$ in each direction) and has a Gaussian transverse profile. The plasma is $0.75 \mathrm{~cm}$ long which corresponds to more than 5 Rayleigh lengths.

\section{PHENOMENOLOGICAL THEORY FOR LWFA IN THE BLOWOUT REGIME AND SIMULATION VERIFICATION}

We next develop a phenomenological theory for LWFA in the blowout regime. A light pulse can be guided with a nearly constant (matched) spot size by a plasma channel that has a parabolic refractive index/density profile with a maximum/minimum on axis. The index of refraction in a plasma can be expanded as [26,27] $\eta=c k / \omega \simeq 1-\frac{1}{2} \times$ $\frac{\omega_{p}^{2}}{\omega_{0}^{2}}\left(1+\frac{\Delta n_{c}}{n_{p}} \frac{r^{2}}{w_{0}^{2}}+\frac{\Delta n}{n_{p}}-\frac{a_{0}^{2}}{8}\right)$, where $\Delta n_{c}$ parametrizes an external density channel, $\Delta n$ is a density depletion from the transverse ponderomotive force, and the term $a_{0}^{2} / 8$ is due to relativistic mass corrections. The characteristic density change required to optically guide such a profile with little spot size oscillation is $\Delta n_{c}=1 /\left(\pi r_{e} w_{0}^{2}\right)$ [26], where $r_{e}=$ $e^{2} / m c^{2}$ is the classical electron radius and $w_{0}$ is the laser spot size. If the density depression is normalized to the plasma density $n_{p}$, this condition becomes $\Delta n_{c} / n_{p} \simeq$ $4 /\left(k_{p} w_{0}\right)^{2}$. An equivalent change to the index of refraction from relativistic mass corrections can also self-guide a laser if $a_{0}^{2} / 8 \gtrsim 4 /\left(k_{p} w_{0}\right)^{2}$ or $P \gtrsim P_{c}$, where $P_{c}=$ $17 \omega_{0}^{2} / \omega_{p}^{2}[\mathrm{GW}]$ is the critical power for relativistic selffocusing [3].

Unfortunately, at the front of the laser, the density compression from the ponderomotive force exerted by the leading edge of the laser causes a decrease to the index of refraction which almost identically cancels the increase from the relativistic mass effects. Therefore, even when $P \gtrsim P_{c}$, the front of the laser is not guided because the index of refraction requires a time $\sim \omega_{p}^{-1}$ to build up. As a consequence, it is often thought that a short pulse laser $\tau \lesssim$ $1 / \omega_{p}$ cannot be self-guided and some form of external optical guiding is needed [26]. However, as described in Ref. [28] for $P / P_{c} \gg 1$, a degree of self-guiding for short pulses is possible because the leading edge of the laser locally pump depletes before it diffracts and the back of the pulse is still guided in the ion column region. In the case of external optical guiding $P / P_{c} \gg 1$ is not necessary, however, if $P / P_{c} \gtrsim 1$ and an appropriate spot size is chosen then clear blowout still occurs. Recently, others have found under certain conditions that ultrahigh power lasers can be self-guided over a few Rayleigh lengths even when the intensity is small enough (and the spot size is large enough) such that a linear (no blowout) wake is excited $[29,30]$. However, in the linear regime for LWFA the focusing and accelerating fields are not ideal and it is difficult to obtain high efficiency. In this recent work test electrons were accelerated and the code did not allow for self-consistent beam loading effects.

The stable self-guiding of an intense short pulse is illustrated in Fig. 1. We find that, for self-guided propagation of the laser beam, without significant variations of the pulse profile over the interaction distance, its spot size and intensity must be appropriately chosen. A rigorous derivation for the matched spot size and the laser profile is not currently available. Still, as noted in Refs. [3,20,21] the requirements for a matched profile can be estimated by assuming that the transverse ponderomotive force of the laser $k_{p} \nabla a_{0}^{2} / \gamma \sim a_{0} /\left(k_{p} R\right)$, where $R$ is the blowout radius, is roughly balanced by the force of the ion channel $E_{r} \sim$ $k_{p} R$, which pulls back the ponderomotively expelled electrons. Equating these two expressions yields $k_{p} R \sim \sqrt{a_{0}}$. Furthermore, the blowout radius scales with the laser spot size, $k_{p} w_{0} \sim k_{p} R$. Through simulations we have found that a more refined condition which leads to only slight oscillations in the spot size is

$$
k_{p} R \simeq k_{p} w_{0}=2 \sqrt{a_{0}} .
$$

We find that this relationship still holds for $a_{0} \gtrsim 2$. In the work of Gordienko and Pukhov [24] it was assumed $k_{p} R \simeq$ $k_{p} w_{0}=1.12 \sqrt{a_{0}}$ (we rewrote this for a linearly polarized laser). We can use Eq. (1) to reformulate the matched beam spot size condition as

$$
a_{0} \simeq 2\left(P / P_{c}\right)^{1 / 3}
$$

As alluded to earlier, the laser etches back due to local pump depletion. In Ref. [28], an estimate of the etching rate, i.e., the etching velocity, based on nonlinear $1 \mathrm{D}$ effects was given as $v_{\text {etch }} \simeq c \omega_{p}^{2} / \omega_{0}^{2}$; and it was verified in 2D PIC simulations. We find that on average this estimate also agrees very well with the observations in numerous 3D PIC simulations. Therefore, the laser will be depleted after a distance (pump depletion length)

$$
L_{\text {etch }} \simeq \frac{c}{v_{\text {etch }}} c \tau_{\text {FWHM }} \simeq \frac{\omega_{p}^{2}}{\omega_{0}^{2}} c \tau_{\text {FWHM }} .
$$

The front of the laser that excites the wake moves backward as the pulse etches back with $v_{\text {etch }}$. The phase velocity 

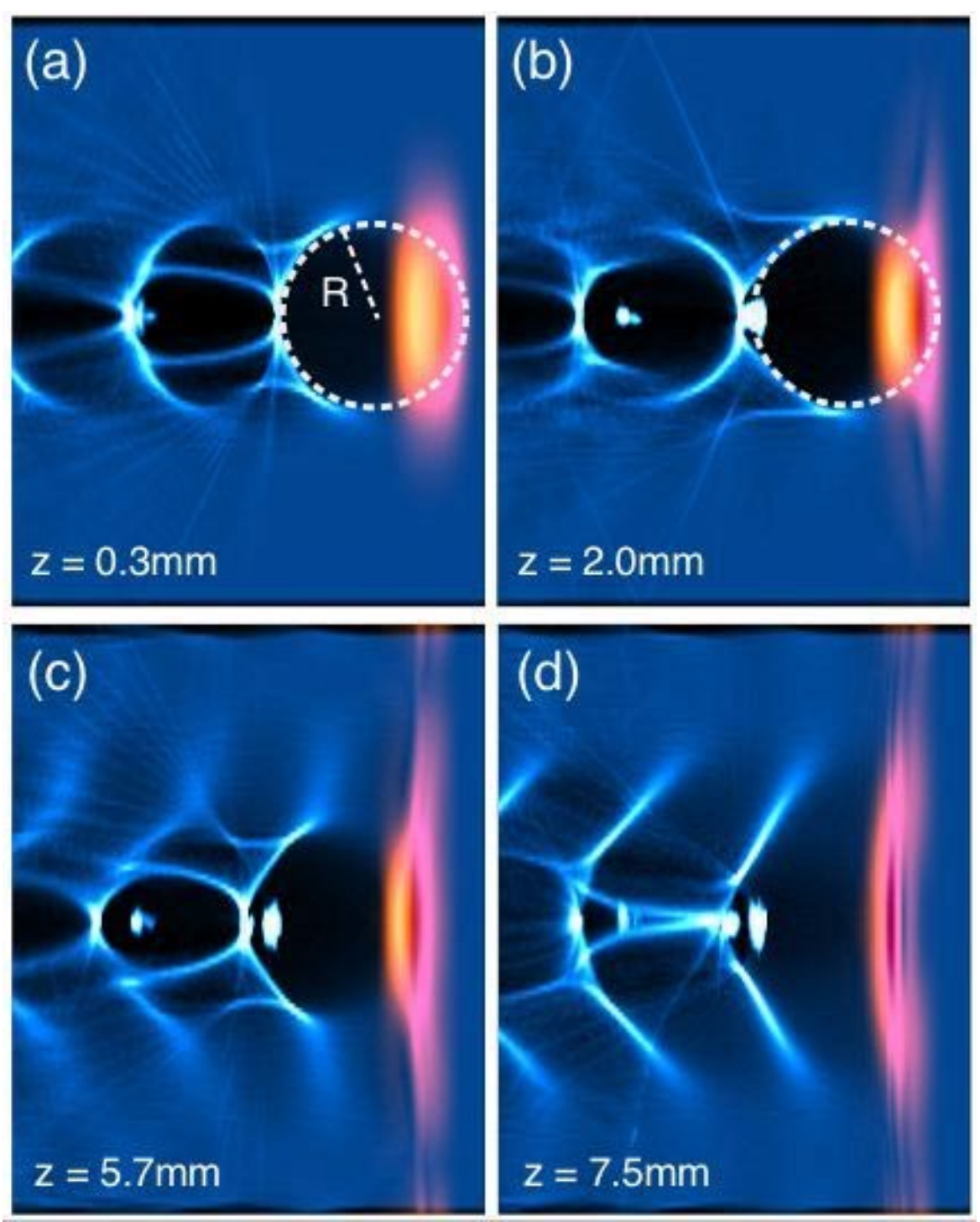

FIG. 1. (Color) A sequence of 2-dimensional slices $(x-z)$ reveals the evolution of the accelerating structure (electron density, blue) and the laser pulse (orange). Each plot is a rectangular of size $z=101.7 \mu \mathrm{m}$ (longitudinal direction, $z$ ) and $x=129.3 \mu \mathrm{m}$ (transverse direction, $x$ ). A broken white circle is superimposed on each plot to show the shape of the blown-out region. When the front of the laser has propagated a distance (a) $z=0.3 \mathrm{~mm}$, the matched laser pulse has clearly excited a wakefield. Apart from some local modification due to beam loading effects, as seen in (b) this wakefield remains robust even as the laser beam propagates though the plasma a distance of $7.5 \mathrm{~mm}$ [as seen in (c) and (d)] or 5 Rayleigh lengths. After the laser beam has propagated $2 \mathrm{~mm}$ [as seen in (b)] into the plasma, one can clearly see self-trapped electrons in the first accelerating bucket. The radial and longitudinal localization of the selftrapped bunch is evident in part (c). After $7.5 \mathrm{~mm}$ the acceleration process terminates as the depleted laser pulse starts diffracting.

of the wake can therefore be expressed as $v_{\phi} \simeq v_{g}-v_{\text {etch }}$, where $v_{g}$ is the linear group velocity of light in a very underdense plasma $\omega_{p}^{2} \ll \omega_{0}^{2} ;$ therefore $v_{\phi} \simeq c[1-$ $\left.3 \omega_{p}^{2} /\left(2 \omega_{0}^{2}\right)\right]$. The fact that the phase velocity of a wake excited by an intense laser was less than even the linear group velocity of a laser was first discussed in [31]. The distance that the trapped electrons travel until they outrun the wave (dephasing length) is

$$
L_{d} \simeq \frac{c}{c-v_{\phi}} R \simeq \frac{2}{3} \frac{\omega_{0}^{2}}{\omega_{p}^{2}} R
$$

We find in numerous 1D, 2D, and 3D simulations that the etching velocity and hence this dephasing estimate works well for $2 \lesssim a_{0} \lesssim 2 \sqrt{\frac{n_{c}}{n_{0}}}$. The estimate for the upper value of $a_{0}$ is discussed later.

To illustrate the process of local pump depletion and its relationship to photon deceleration [27], we plot the wave number, $k_{z}(z)$, of one component of the laser's electric field $\left(E_{x}\right)$ after it has propagated through $0.18 \mathrm{~cm}$ of plasma for three different laser intensities, $a_{0}=1,4,10$ in Fig. 2. For the corresponding simulations in Figs. 2(a) and 2(b), $a_{0}=$ 4 but the pulse length was 30 and $50 \mathrm{fs}$, respectively. For 

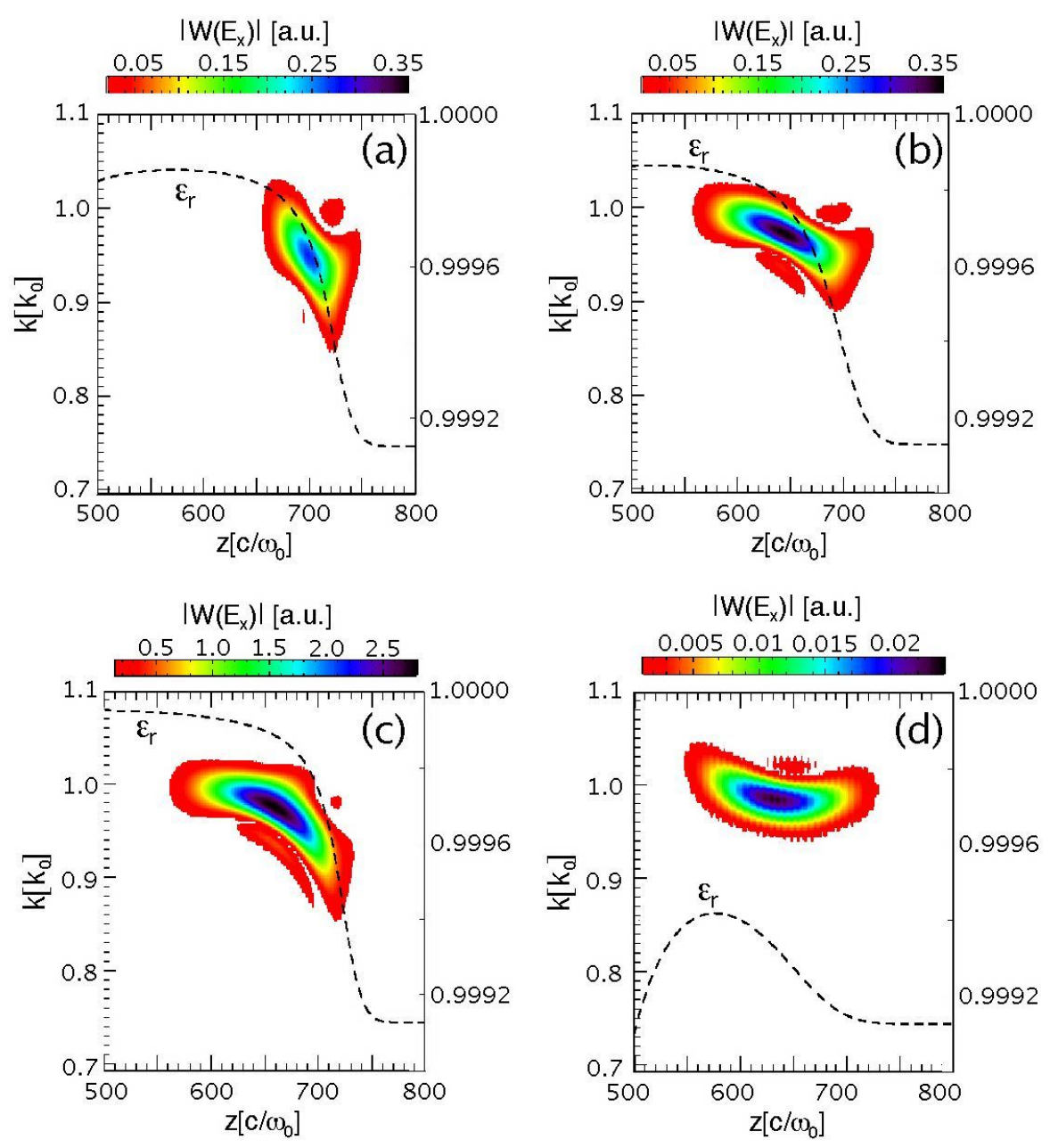

FIG. 2. (Color) Four plots depicting the Wigner transform of the $x$ component of the laser electric field in the middle of the simulation box and the relative permittivity as functions of $z$. The Wigner transform of a function $\varphi(z)$ is defined as [39] $W_{\varphi}(z, k)=$ $\int_{-\infty}^{\infty} e^{-i k z^{\prime}} \varphi\left(z+\frac{z^{\prime}}{2}\right) \varphi^{*}\left(z-\frac{z^{\prime}}{2}\right) d z^{\prime}$ and the relative permittivity valid under the quasistatic approximation is $\varepsilon_{r}=\eta^{2}=1-\frac{n_{p}}{n_{c}} \frac{1}{1+\psi}$. In each plot, the vertical axis on the left corresponds to the local wave number over the initial laser wave number and the vertical axis on the right to $\varepsilon_{r}$. The four plots represent results from four different computer simulations after $0.18 \mathrm{~cm}$ of laser propagation into a plasma with $n_{p}=1.5 \times 10^{18} \mathrm{~cm}^{-3}$. (a) A 3D simulation of a $30 \mathrm{fs}$ laser with $a_{0}=4$ and matched profile. (b) A 2D simulation of a 50 fs laser with $a_{0}=4$ and matched profile. (c) A 2D simulation of a 50 fs laser with $a_{0}=10$ and matched profile. (d) A 2D simulation of a 50 fs laser with $a_{0}=1$. Plot (c) is typical for the ultrarelativistic blowout regime while plot (d) is typical for the linear regime. Plots (a) and (b), which result from simulations with $a_{0}=4$, are more similar to the ultrarelativistic blowout case.

the other two cases, the pulse length was $50 \mathrm{fs}$. In each case the plasma density was $1.5 \times 10^{18} \mathrm{~cm}^{-3}$. The laser spot size was matched according to Eq. (1) for the first three cases and was $24 \mu \mathrm{m}$ for the case (d). The leading edge of the laser does work by pushing electrons forward and outward, and the index of refraction is modified by the combined effects of the electron density decrease and the relativistic mass increase. One way to view pump depletion is that the gradient of the index of refraction leads to a decrease in the laser frequency (photon deceleration) and, because the number of photons is an invariant, a depletion to the laser's energy [27]. As the laser intensity is increased this gradient becomes steeper and more localized to the front of the laser. This is seen in Fig. 2, where for $a_{0}=1$ the spectra has a $U$ shape [32] and the very front is not absorbed, while for $a_{0}=10$ only the front decreases and the back is not effected. A chirp in wave number (frequency) can lead to pulse compression since the faster photons in the tail catch the slower photons in the head of the beam [32-35]. However, in nonlinear wakes the electron density on axis is nearly zero so the relative phase slippage between the photons with different frequency will be less. In nonlinear wakes the front of the laser can also be absorbed before it diffracts [28]. The spectra for $a_{0}=4$ are much more similar to the $a_{0}=10$ case than to the $a_{0}=1$ case. In Ref. [28] the scaling for the etching rate was also obtained from the energy given to the plasma electrons from a laser. Deriving a more rigorous theory for the phase 
velocity of the wake in the blowout regime and speeding it up by modifying the initial pulse shape and frequency spectra are areas for future work.

For the energy gain we may write the obvious equation

$$
\Delta E=q E_{\mathrm{LW}} L_{\mathrm{acc}}=\epsilon_{\mathrm{LW}} l_{\mathrm{acc}} m c^{2},
$$

where $E_{\mathrm{LW}}$ is the average accelerating field of the beam loaded wake, $L_{\text {acc }}$ is the acceleration length, $\epsilon_{\mathrm{LW}} \equiv$ $e E_{\mathrm{LW}} /\left(m c \omega_{p}\right)$ and $l_{\mathrm{acc}} \equiv \omega_{p} L_{\mathrm{acc}} / c$. The desired acceleration length is the dephasing length, so we impose the condition $L_{\text {etch }}>L_{d} \Rightarrow c \tau_{\mathrm{FWHM}}>2 R / 3$. If the pulse is too short dephasing will not be reached, and the electron beam may have significant energy spread. We note that this condition is approximate, since the laser starts diffracting as soon as its intensity is insufficient to sustain selffocusing and this happens before the pulse is completely pump depleted. Additionally, the injected particles need to slightly pass the dephasing point (phase space rotation) so that the energy spread is minimum. On the other hand, the length of the pulse should not be too large, because the laser field could interact with the trapped electrons and degrade the beam quality $[7,13]$.

We need an expression for $\epsilon_{\mathrm{LW}}$ which is the average accelerating field experienced by an electron, $\left\langle E_{z}\right\rangle$. In Ref. [21] it was shown that for $a_{0} \geqslant 4$ the ion column formed a sphere and that the accelerating field, for the most part, depends linearly on the distance from the middle of the sphere [Fig. 3(a)]. This is confirmed in Figs. 1(a)-1(c), where a bubble void of electrons roughly forms a circle and in Figs. 3(a) and 3(b) where a lineout of $e E_{z} /\left(m c \omega_{p}\right)$ along the axis is shown. We also found by theory and 3D PIC simulations that a spherical shape is still roughly formed for $2 \lessgtr a_{0} \lesssim 4$ with matched laser profiles. Interestingly, although the physics is very different, the nonlinear wakes formed in 1D also exhibit a linear slope of the same amount.

Because the bubble is roughly a sphere and the electrons are either self-injected or externally injected at the rear, the electrons then travel a relative distance $R$ before they dephase. The peak useful accelerating field is $e E_{z, \max } /\left(m c \omega_{p}\right)=\sqrt{a_{0}}$ and because the wakefield is roughly linear, the average field is half of the peak: $\epsilon_{\mathrm{LW}} \equiv$ $e E_{z, \max } /\left(2 m c \omega_{p}\right) \simeq \sqrt{a_{0}} / 2$. We can therefore write the approximate equation for the energy gain:

$$
\begin{aligned}
\Delta E & \simeq \frac{2}{3} m c^{2}\left(\frac{\omega_{0}}{\omega_{p}}\right)^{2} a_{0} \simeq m c^{2}\left(\frac{P}{m^{2} c^{5} / e^{2}}\right)^{1 / 3}\left(\frac{n_{c}}{n_{p}}\right)^{2 / 3} \\
\Delta E[\mathrm{GeV}] & \simeq 1.7\left(\frac{P[\mathrm{TW}]}{100}\right)^{1 / 3}\left(\frac{10^{18}}{n_{p}\left[\mathrm{~cm}^{-3}\right]}\right)^{2 / 3}\left(\frac{0.8}{\lambda_{0}[\mu \mathrm{m}]}\right)^{4 / 3} .
\end{aligned}
$$

We emphasize the much stronger dependence of the beam energy on the plasma density than on the input laser power. However, when the plasma density is lowered for fixed power, ensuring self-guided propagation of the lead-
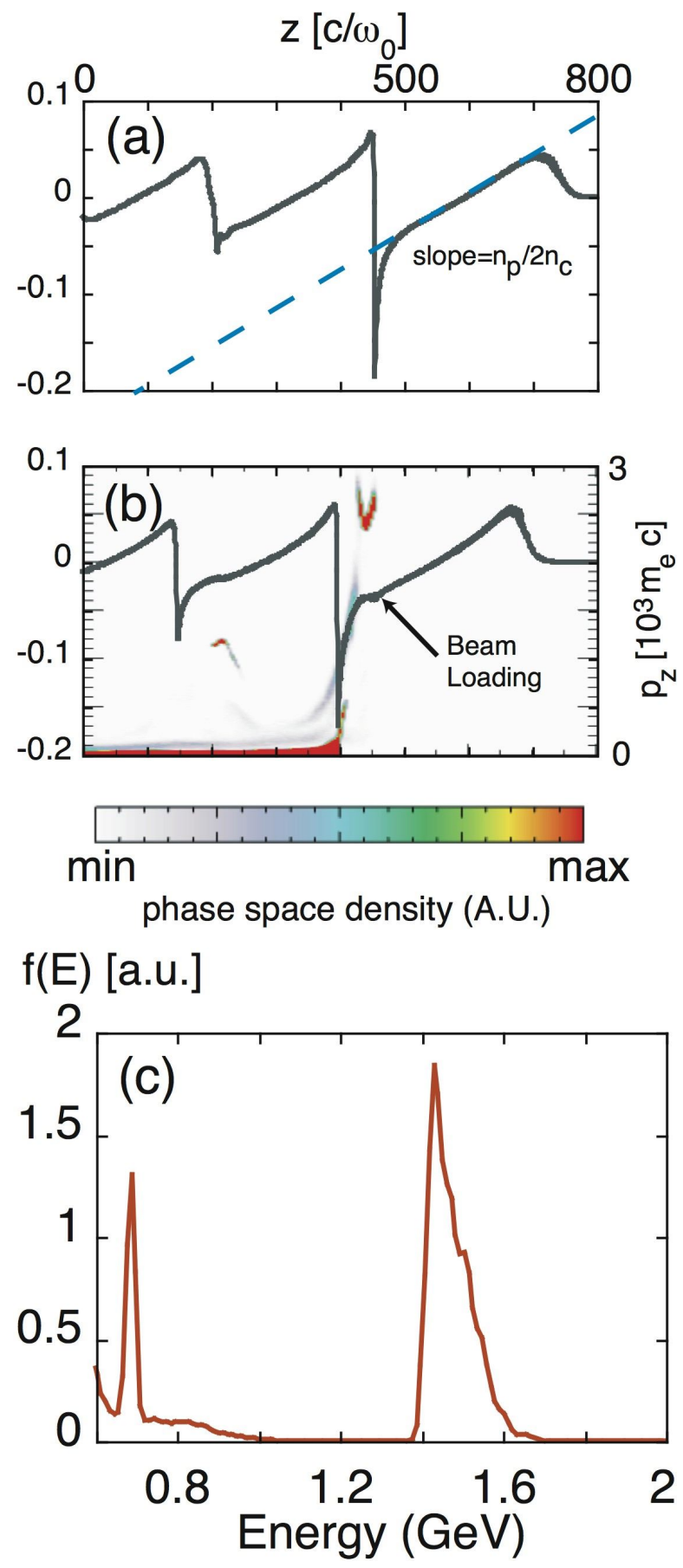

FIG. 3. (Color) (a) A lineout of the wakefield along the $z$ axis after $0.3 \mathrm{~mm}$ shows that within the first bucket the slope of the wakefield is nearly constant and equal to $e E_{z} /\left(m c \omega_{p}\right) \simeq \xi / 2$, where $\xi=\left[k_{p}(c t-z)\right]$. After $5.7 \mathrm{~mm}$ of propagation (b) the wakefield has been modified by beam loading (flattening of the wake between $\left.400-450 c / \omega_{p}\right)$. This is corroborated by the $p_{z}$ vs $z$ plot that is superimposed on the lineout of the wakefield. Panels (a) and (b) reveal that the acceleration mechanism is extremely stable during the simulation. The energy spectrum after $7.5 \mathrm{~mm}$ (c) exhibits an isolated spike of $0.3 \mathrm{nC}$ at $1.5 \mathrm{GeV}$ with energy spread $\Delta \gamma / \gamma=3.8 \%$ corresponding to the first bucket and a second spike of $50 \mathrm{pC}$ at $700 \mathrm{MeV}$ with energy spread $\Delta \gamma / \gamma=1.5 \%$ corresponding to the second bucket. 
ing edge of the laser is more challenging. This can be accomplished by plasma channels or to some degree by self-guiding. As we argue later, for self-guiding to occur $P / P_{c}$ needs to increase as the plasma density decreases. We can rewrite Eq. (6) in terms of the critical power for relativistic self-focusing, $P_{c}$ :

$$
\Delta E[\mathrm{GeV}] \simeq 3.8\left(\frac{P}{P_{c}}\right)^{-2 / 3} \frac{P[\mathrm{TW}]}{100} .
$$

On the other hand, if a plasma channel is used, $P / P_{c}$ can be kept as low as 1 . As shown before, the channel depth $\Delta n_{c} / n_{p}$ needed is $4 /\left(k_{p} w_{0}\right)^{2} \simeq 1 / a_{0}$. So as long as $a_{0} \geqslant$ 2 is used, the normalized channel depth is small (less than 0.5 ). It is also worth noting here that, when a channel is used, the channel parameters (width and depth) should be chosen based on the matching condition for given laser power and plasma density [Eqs. (1) and (2)] so that the leading front of the laser is guided by the density channel and the back of the laser is guided by the matched ion channel.

The electrons which are accelerated can be either selfinjected as shown in our sample simulation [Fig. 1(b)] or externally injected from some other source. For selfinjection, particles in the rear of the blowout region must be able to catch up with the wake. The physical condition for this to happen is twofold: first, the blowout radius should be large enough so that, when the particles reach the rear of the bubble, they move predominately in the forward direction with speed close to the speed of light. Second, at the rear portion of the ion channel, trajectory crossing occurs leading to a narrow sheath with the highest accelerating and focusing fields. Therefore, even though electrons initially have a $\gamma$ (energy) substantially below the wake's Lorentz factor $\gamma_{\phi}$, they can easily achieve suffi- cient energy as they are accelerated while they slowly drift backwards (relatively to the pulse) in the sheath. In our sample simulation, the effective $\gamma_{\phi}$ of the wake is around 20 and the normalized blowout radius is around 4. The initial energy $\gamma$ of those trapped electrons is substantially smaller than 20. For even lower plasma densities, we have performed a number of simulations, where an electron beam with $\gamma$ exceeding 10000 was used as the driver instead of a laser and we observed self-injected electrons in each case for a normalized blowout radius around 5 . This indicates that for laser wavelengths in the $0.8 \mu \mathrm{m}$ range and plasma densities of interest, self-injection will always happen when we keep the normalized blowout radius around $4-5$. This differs significantly from the claim in Ref. [20] that $\sqrt{a_{0}}>\gamma_{\phi}$, or $a_{0}>400$ for our sample simulation, for self-trapping to occur.

In the regime presented here, the self-injected electron bunches are highly localized in space with a half-width of the first bunch of only $\sim 10 \mathrm{fs}$, i.e. $1 c / \omega_{p}$. Once a sufficient number of electrons have been trapped the trapping process terminates, as seen in Fig. 1(c). The first electron bunch reaches an energy of $1.5 \mathrm{GeV}$ and its energy spectrum is presented in Fig. 3(c). The normalized emittances are shown in Fig. 4. They may be estimated as the product of the beam spot size, which roughly scales with $1 / \sqrt{n_{p}}$, with the spread in the momentum perpendicular to the acceleration direction, which scales with the relativistic ponderomotive potential $\left(\sim a_{0}\right)$. These simple considerations show that as we move to lower densities in order to achieve higher-energy particles the emittances of the self-injected electrons will increase. This suggests that, for the electron beam to be useful for high-energy physics or light source, external injection may be more attractive. As an interesting aside, simulations also reveal the trapping and acceleration

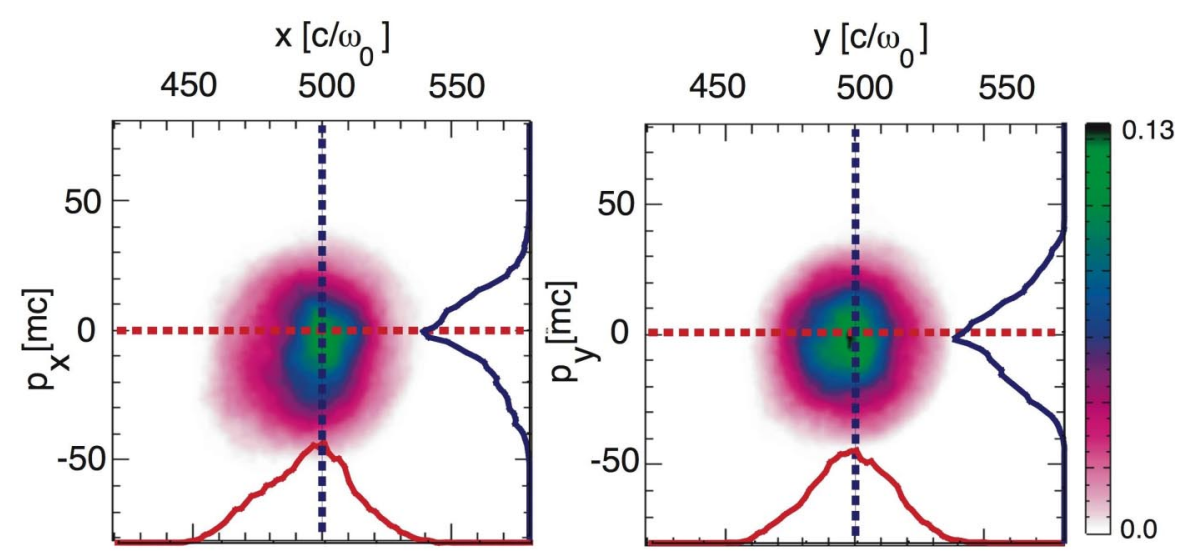

FIG. 4. (Color) The normalized emittance $\left[\varepsilon_{N}\right]_{i}=\pi \sqrt{\left\langle\Delta p_{i}^{2}\right\rangle\left\langle\Delta x_{i}^{2}\right\rangle-\left\langle\Delta p_{i} \Delta x_{i}\right\rangle^{2}}$ (where $\Delta p_{i}$ is normalized as indicated by the figure and the emittance is in units of $\Delta x_{i}$ ) and is the approximate area in phase space $p_{i} x_{i}$. For the panels above which correspond to the first bunch, this formula yields $\left[\varepsilon_{N}\right]_{x} \simeq 35 \pi \mathrm{mm} \mathrm{rad}$ and $\left[\varepsilon_{N}\right]_{y} \simeq 29 \pi \mathrm{mm} \mathrm{rad}$. An upper limit for the emittance can be found by multiplying the typical divergences shown in the figure; this method leads to an overestimation which for this case is about $25 \%$. For the second bunch of accelerated electrons (not shown in this figure), the emittances are significantly lower: $\left[\varepsilon_{N}\right]_{x} \simeq 10 \pi \mathrm{mm}$ rad and $\left[\varepsilon_{N}\right]_{y} \simeq 11 \pi \mathrm{mm} \mathrm{rad}$. 
of a second distinct bunch in the second bucket [see Fig. 3(c)], which has a lower energy because the average electric field it experiences is smaller.

The number, $N$, of electrons that are accelerated can be estimated from energy balance. Hence, we examine the partition of field and particle energy within the first bucket. The fields inside the ion column have $E_{z}, E_{r}$, and $B_{\phi}$ components. In addition there is kinetic energy in the plasma. In a 3D linear or 1D nonlinear wake the fields and kinetic energy scale together. This is not the case for these 3D nonlinear wakes where an increasing percentage of energy ends up in electrons which are blown out well beyond the narrow electron sheath for higher laser intensity. Integrating the field energy in the ion channel we find equipartition between the energy in the longitudinal field $\mathcal{E}_{l}$ and the focusing fields $\mathcal{E}_{f}$ :

$$
\mathcal{E}_{l} \simeq \mathcal{E}_{f} \simeq \frac{1}{2} \mathcal{E}=\frac{1}{120}\left(k_{p} R\right)^{5}\left(\frac{m^{2} c^{5}}{e^{2} \omega_{p}}\right)
$$

The trailing particles can recover the field energy in the ion channel and the kinetic energy in the narrow electron sheath by changing the shape of the ion channel. The kinetic energy in the narrow electron sheath scales the same way as the field energy. By equating $\mathcal{E}$ with the energy absorbed by $N$ particles that travel across the ion channel (we assume the average field felt by these particles is $E_{z, \max } / 2$ and the kinetic energy in the electron sheath absorbed by the trailing particles is the same as the field energy), we obtain

$$
N \simeq \frac{1}{30}\left(k_{p} R\right)^{3} \frac{1}{k_{p} r_{e}}=\left(\frac{\beta^{3}}{\alpha}\right) \frac{8 / 15}{k_{0} r_{e}} \sqrt{\frac{P}{m^{2} c^{5} / e^{2}}},
$$

where $\alpha=k_{p} w_{0} /\left(2 \sqrt{a_{0}}\right)$ and $\beta=k_{p} R /\left(2 \sqrt{a_{0}}\right)$. Using Eq. (1) $\alpha \simeq 1 \simeq \beta$ we obtain

$$
N \simeq \frac{8 / 15}{k_{0} r_{e}} \sqrt{\frac{P}{m^{2} c^{5} / e^{2}}} \simeq 2.5 \times 10^{9} \frac{\lambda_{0}[\mu \mathrm{m}]}{0.8} \sqrt{\frac{P[\mathrm{TW}]}{100}} .
$$

The efficiency scales as the total energy $\mathcal{E}_{b}$ in the accelerated electron beam [energy gain Eq. (6) times particle number from Eq. (9)] divided by the total laser energy $\mathcal{E}_{T}$ (assuming $c \tau \simeq 2 \sqrt{a} c / \omega_{p} 0$ ):

$$
\Gamma \sim \mathcal{E}_{b} / \mathcal{E}_{T} \sim 1 / a_{0}
$$

which indicates that $a_{0}$, i.e., $\left(P / P_{c}\right)^{1 / 3}$ cannot be too large if one needs high efficiency. For a $200 \mathrm{TW}, 0.8 \mu \mathrm{m}$ pulse, Eq. (10) predicts $0.6 \mathrm{nC}$ of charge. The charge measured from the simulation for the first bunch is $0.3 \mathrm{nC}$. We have also verified these scaling laws by monitoring how many electrons can be externally injected before the wake becomes severely loaded.

As shown in Fig. 1(d), the acceleration process stops before the accelerating bunch dephases. This will not lead to any considerable modifications of the aforementioned formulas, particularly because the pump depletion length scales as the dephasing distance and the accelerating wakefield decreases as the trapped electrons approach the center of the sphere.

The beam energy seen in the simulation, $1.5 \mathrm{GeV}$, is close to that calculated theoretically from Eq. (6) which is (6) $\Rightarrow \Delta W \simeq 1.6 \mathrm{GeV}$. Using formulas (3) and (4), we see $L_{d} \simeq 1.31 \mathrm{~cm}>0.96 \mathrm{~cm} \simeq L_{p d}$ which is in agreement with our observation that pump depletion happened before dephasing. In addition, as the laser pump depleted its rate of diffraction increased such that the effective acceleration length was eventually limited by diffraction to a distance $0.75 \mathrm{~cm}$ which was less than the pump depletion distance $0.96 \mathrm{~cm}$. For a wake with a linear slope most of the energy gain occurs near the peak field. This combined with the density spike at the back of the bucket is why the estimate from Eq. (6) is still so accurate for this nonoptimized sample simulation.

In spite of the complexity of the physics associated with this interaction, the predictions by the simple formulas presented in this article are very close to 3D PIC simulation results. Good agreement is also achieved between these scaling laws and recent experimental results [10-12] despite the fact that the laser powers were slightly below the "threshold" for the blowout regime and the laser pulse was not matched transversely nor longitudinally. To be in the regime identified by a spherical bubble, one needs $a_{0} \geqslant 4$ or equivalently $P / P_{c} \geqq 8$ and $c \tau<2 \sqrt{a_{0}} c / \omega_{p}$ which leads to the condition that $P \gtrsim 30(\tau / 30 \mathrm{fs}) \mathrm{TW}$. These conditions can be relaxed for channel-guided lasers for which $P / P_{c}$ may be smaller. It is then written as $a_{0} \gtrsim 2$ or $P / P_{c} \gtrsim 1$. We present the comparison between the scaling law for the energy Eq. (6) and the aforementioned results in Fig. 5.

The scalings derived above and the underlying physics predict that it is advantageous to use moderate intensities and very low plasma densities to increase the output energy and keep the efficiency high. The simulations also show that the injection process can be clamped and the energy spread of the electron beam is much less in the regime we are proposing, which indicates that this regime is also amenable to accelerating externally injected beams while maintaining good beam quality.

In Table I we compare some of our results to previously published work [24,26]. Both linear and 1D nonlinear formulas fail to describe the 3D nonlinear regime in nearly all respects. It is however instructive to compare the scaling laws described above against those obtained in Ref. [24] using a similarity theory for the high intensity high plasma density regime, because in Ref. [24] the scaling (but not the coefficients) of the fields in the bubble and the laser spot size with $a_{0}$ were identified correctly. The key assumption of Ref. [24] required that $a_{0} \gg 1$. For the energy gain, they obtained 


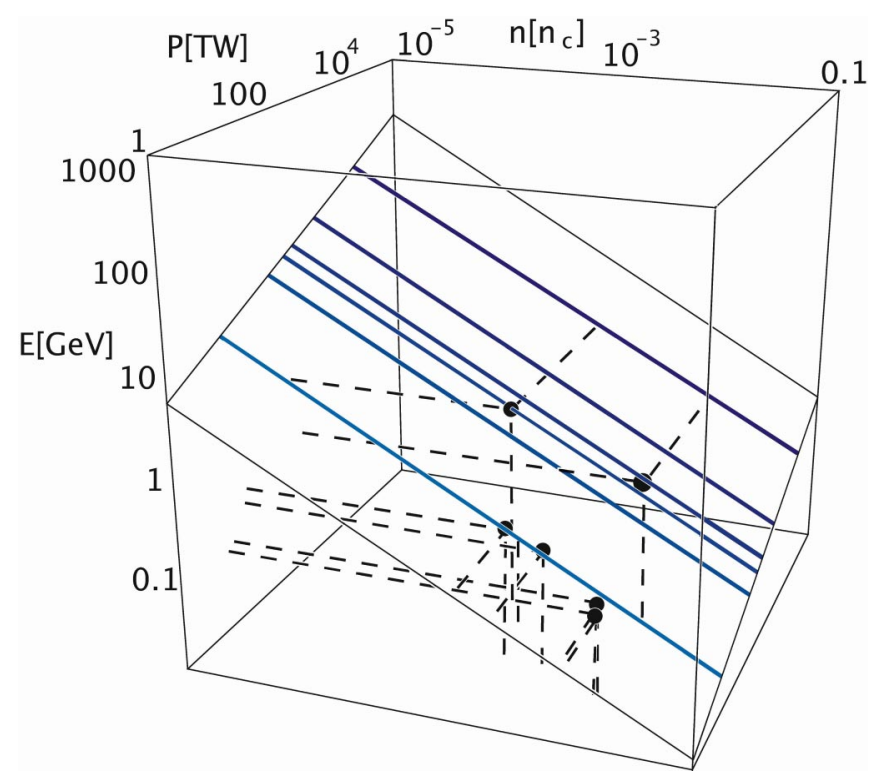

FIG. 5. (Color) $E[\mathrm{GeV}]$ vs power $P[\mathrm{TW}]$ and density $n / n_{c}$ from Eq. (6): The blue lines of constant power show the strong dependence of the energy of the self-trapped electrons to the density. The black points correspond to (a) experiment [10], (b) experiment [11] which uses a channel for guiding, (c) experiment and 3D PIC simulation [12], (d) 3D PIC simulation [7] which uses a channel for guiding, (e) A 3D PIC simulation in [6], and (f) 3D PIC simulation presented in this article. Each of these points is very close to 1 of the blue lines indicating agreement with our scaling law.

$$
E_{\mathrm{mono}} \approx 0.65 m c^{2} \sqrt{\frac{P}{m^{2} c^{5} / e^{2}}} \frac{c \tau}{\lambda}
$$

This formula also implies a dependence on plasma density if $c \tau \approx w_{0} \approx \sqrt{a_{0}} c / \omega_{p}$ is assumed. Under these optimum conditions, formula (12) can be rewritten as

$$
E_{\mathrm{mono}} \approx 0.16 m c^{2} \frac{c \tau}{w_{0}}\left(\frac{P}{m^{2} c^{5} / e^{2}}\right)^{2 / 3}\left(\frac{n_{c}}{n_{p}}\right)^{1 / 3} .
$$

We point out the stronger dependence of the energy on the power in these formulas. The difference between the scaling laws presented here and those from similarity theory lie in the scaling for $L_{\text {acc }}$. We have argued that $L_{\text {acc }}$ is limited because the pulse pump depletes primarily by giving kinetic energy to the electrons. An electron at the front of the laser is pushed forward and to the side. As argued in Ref. [28] each electron gains an amount of energy that scales as $\left(a_{0}^{2} / 2\right) m c^{2}$. Since the laser's energy also scales as $a_{0}^{2}$, this " $1 \mathrm{D}$ like" pump depletion enhances the etching velocity independently of laser intensity. However, for extremely large $a_{0}$ electrons can move forward with a velocity greater than the velocity of the leading edge of the pulse, which is at most the linear group velocity because the nonlinearity does not develop instantaneously. Equating the forward going velocity of a single electron to the linear group velocity gives an estimate for a critical value for the laser amplitude, $a_{0 c} \approx 2 \sqrt{n_{c} / n_{p}}$. We can see that for high plasma density $n_{p} / n_{c} \approx 0.01-0.08$ the critical value for $a_{0}$ is $a_{0 c} \approx 20-8$, but for lower plasma densities $n_{p} / n_{c} \sim 0.001, a_{0 c} \geq 70$.

For laser amplitudes above this critical value, the laser will pump deplete more slowly because the kinetic energy given to each electron will no longer scale as $a_{0}^{2}$. In this limit a constant percentage of the energy may go into the

TABLE I. This table compares formulas and scalings from linear theory, 1D nonlinear theory, the similarity theory of Ref. [24] and the 3D phenomenological nonlinear theory in this paper. The column labeled $a_{0}$ shows the range of $a_{0}$ that each theory is intended for. $k_{p} w_{0}$ from linear and 1D nonlinear theory is kept constant while in both our theory and the theory of Ref. [24]. $k_{p} w_{0}$ scales with the blowout radius. The acceleration length is limited by dephasing in linear theory and our 3D nonlinear theory, while it is limited by pump depletion in 1D nonlinear theory and the work of GP. (Note that in standard 1D nonlinear theory the phase velocity of the wake is incorrectly set equal to the nonlinear group velocity.) Only in our 3D nonlinear theory do dephasing and pump depletion scale the same way (if the pulse length is matched to the blowout radius). The dephasing length is evidently ignored in Ref. [24] so the energy gain depends on the pulse length. Linear dephasing and 1D nonlinear dephasing both scale differently than in the 3D nonlinear regime. The pump depletion length from 1D nonlinear theory scales as in 3D nonlinear theory but for different physics reasons. In 1D, all of the laser energy goes into the wake while in our 3D theory this is not the case. We discuss the pump depletion length from Ref. [24] extensively in the text. Both linear and 1D nonlinear theory incorrectly identify the plasma wavelength and wake phase velocity. The resulting energy scalings are different. If one sets $\omega_{p} \tau \sim \sqrt{a_{0}}$ in Ref. [24], then the energy scales as $a_{0}^{2}$. Therefore linear, 1D nonlinear, and the similarity theory of Ref. [24] all give identical scalings for the $\Delta W$ with both intensity and density while in the regime presented here $\Delta W$ scales as $a_{0}$ to the first power.

\begin{tabular}{lcccccccc}
\hline \hline & $a_{0}$ & $k_{p} w_{0}$ & $\epsilon_{L W}$ & $k_{p} L_{d}$ & $k_{p} L_{p d}$ & $\lambda_{W}$ & $\gamma_{\phi}$ & $\Delta W /\left(m c^{2}\right)$ \\
\hline Linear: & $<1$ & $2 \pi$ & $a_{0}^{2}$ & $\frac{\omega_{0}^{2}}{\omega_{p}^{2}}$ & $\frac{\omega_{0}^{2}}{\omega_{p}^{2}} \frac{\omega_{p} \tau}{a_{0}^{2}}$ & $\frac{2 \pi}{k_{p}}$ & $\frac{\omega_{0}}{\omega_{p}}$ & $a_{0}^{2} \frac{\omega_{0}^{2}}{\omega_{p}^{2}}$ \\
1D Nonlinear: & $>1$ & $2 \pi$ & $a_{0}$ & $4 a_{0}^{2} \frac{\omega_{0}^{2}}{\omega_{p}^{2}}$ & $\frac{1}{3} \frac{\omega_{0}^{2}}{\omega_{p}^{2}} \omega_{p} \tau$ & $\frac{4 a_{0}}{k_{p}}$ & $\sqrt{a_{0}} \frac{\omega_{0}}{\omega_{p}}$ & $4 a_{0}^{2} \frac{\omega_{0}^{2}}{\omega_{p}^{2}}$ \\
3D Nonlinear: & $>2$ & $2 \sqrt{a_{0}}$ & $\frac{1}{2} \sqrt{a_{0}}$ & $\frac{4}{3} \frac{\omega_{0}^{2}}{\omega_{p}^{2}} \sqrt{a_{0}}$ & $\frac{\omega_{0}^{2}}{\omega_{p}^{2}} \omega_{p} \tau$ & $\sqrt{a_{0} \frac{2 \pi}{k_{p}}}$ & $\frac{1}{\sqrt{3}} \frac{\omega_{0}}{\omega_{p}}$ & $\frac{2}{3} \frac{\omega_{0}^{2}}{\omega_{p}^{2}} a_{0}$ \\
Ref. [24]: & $>20$ & $\sqrt{a_{0}}$ & $\sqrt{a_{0}}$ & & $a_{0} \frac{\omega_{0}^{2}}{\omega_{p}^{2}} \omega_{p} \tau$ & & $\frac{\omega_{0}^{2}}{\omega_{p}^{2}} a_{0}^{3 / 2} \omega_{p} \tau$ \\
\hline \hline
\end{tabular}


fields of the wake such that $\Gamma \sim$ constant as predicted by Ref. [24]. The pump depletion length, $L_{p d}$, can then be estimated from energy balance between the laser and the wakefields, $\frac{E_{0}^{2}}{8 \pi} c \tau \approx \frac{E_{z}^{2}}{8 \pi} L_{p d} \Rightarrow L_{p d} \approx \frac{a_{0}^{2}}{\epsilon^{2}} \times$ $\frac{\omega_{0}^{2}}{\omega_{p}^{2}} c \tau \approx \frac{1}{4} a_{0} \frac{\omega_{0}^{2}}{\omega_{p}^{2}} c \tau \sim a_{0} L_{\text {etch }}$ which does depend on $a_{0}$. Using this scaling for $L_{\mathrm{acc}}$ in Eq. (5) provides the same scaling for the particle energy as obtained from similarity theory. However, even for this high intensity limit dephasing will still occur because the leading edge cannot move faster than the linear group velocity of the highest frequency component, since at the very front the amplitude is small [31].

Last, it is useful to extrapolate this nonlinear regime for moderate $a_{0}$ to $10 \mathrm{GeV}$ and beyond. If we keep $P / P_{c}$ fixed, then the laser power and inverse of the density scale with the desired electron energy and the pulse length will scale as the electron energy to the $1 / 2$ power. If we scale up our sample simulation, to obtain $15 \mathrm{GeV}$ we need a $2 \mathrm{PW}$, $100 \mathrm{fs}$ laser and a plasma with density of $1.5 \times 10^{17} \mathrm{~cm}^{-3}$. The only uncertainty is whether self-guiding is still possible.

To obtain the condition for self-guiding, we resort to quasistatic theory where the index of refraction $\eta \simeq 1-$ $\frac{1}{2} \frac{\omega_{p}^{2}}{\omega_{0}^{2}} \frac{1}{1+\psi}$ and $\frac{\partial^{2} \psi}{\partial \xi^{2}}-\frac{k_{p}^{2}}{2}\left[\frac{1+a^{2}}{(1+\psi)^{2}}-1\right]=0$. As noted earlier $1-\frac{1}{1+\psi} \simeq \psi$ must equal $4 /\left(k_{p} w_{0}\right)^{2}$ in order for guiding to occur. For $|\psi| \ll 1$, we assume $\frac{\partial^{2} \psi}{\partial \xi^{2}} \simeq \frac{k_{p}^{2}}{2} a^{2}$; therefore $\psi$ builds up to the necessary value in a distance $\Delta \xi$ which scales as $\Delta \xi \sim\left(k_{p}^{2} w_{0} a_{0}\right)^{-1} \sim\left(k_{p} a_{0}^{3 / 2}\right)^{-1}$. The length of the laser that is lost due to diffraction each Rayleigh length $\left(Z_{R}\right)$ will also scale as $\left(k_{p} a_{0}^{3 / 2}\right)^{-1}$. Self-guiding can be achieved if the length lost due to pump depletion in each Rayleigh length also scales the same way:

$$
\frac{v_{\text {etch }}}{c} \simeq \frac{3}{2} \frac{n_{p}}{n_{c}} \sim \frac{\Delta \xi}{Z_{R}} \Rightarrow a_{0} \sim\left(n_{c} / n_{p}\right)^{1 / 5} .
$$

This scaling law indicates that as the density is decreased to increase the electron energy we need to slowly increase the laser intensity to maintain self-guiding. As a result the 2 PW laser pulse estimate needs to be slightly modified. Instead we choose $P / P_{c} \simeq 28 \Rightarrow a_{0} \simeq 6$, and a density $n=4 \times 10^{17} \mathrm{~cm}^{-3}$. For these parameters stable selfguiding should occur and lead to acceleration of $1.8 \mathrm{nC}$ charge at approximately $9 \mathrm{GeV}$ after $10 \mathrm{~cm}$ of laser propagation. Carrying out a full-scale PIC simulation for the $10 \mathrm{~cm}$ of propagation distance is beyond current capabilities. However, we have carried out a 3D PIC simulation for a short propagation distance to verify that electrons will still be self-injected. Results are shown in Fig. 6.

If both the technology for making meter scale $(0.1 \mathrm{~m} \sim$ $1 \mathrm{~m}$ ) low density plasma channels for guiding and for synchronized external injection [36] are developed, then much higher-energy gain can be achieved for the same laser power. For example, using $P / P_{c} \simeq 1$ (and the channel depth $\Delta n_{c} / n_{p}=0.5$ ), a $10 \mathrm{GeV}$ electron beam with $\sim 0.6 \mathrm{nC}$ charge could be obtained with a $250 \mathrm{TW}, 100 \mathrm{fs}$ laser and a $43 \mathrm{~cm}$ long plasma channel with a minimum density $n=1.2 \times 10^{17} \mathrm{~cm}^{-3}$. Extrapolating the parameters further, we predict that a $120 \mathrm{GeV}$ electron beam with

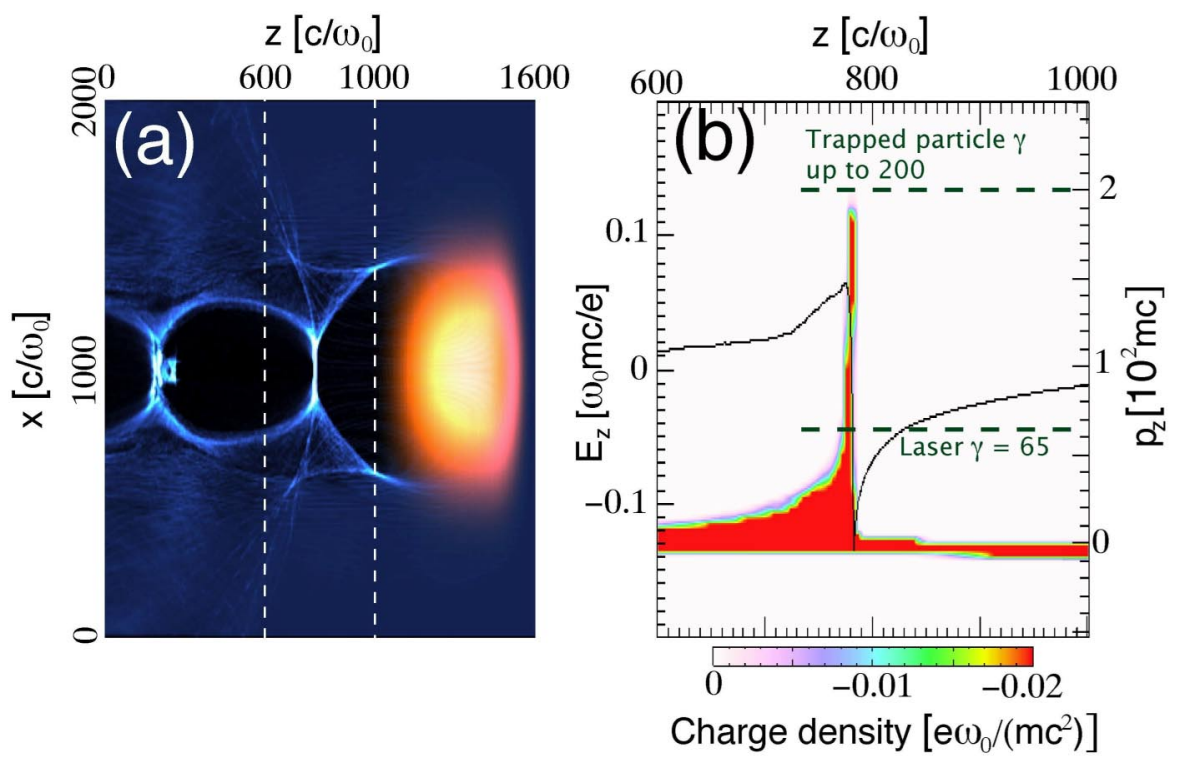

FIG. 6. (Color) Panel (a) shows the laser electric field with orange on top of the electron density with blue after $0.4 \mathrm{~mm}$ of propagation. The laser front is chosen sharper than the back because it has been found through simulations that this leads to more stable propagation. The blowout region is well formed. Panel (b) shows that particles at the rear of the ion channel have already reached velocities higher than the laser velocity and therefore are trapped. The region in the $z$ direction plotted in panel (b) corresponds to the $z$ region between the broken lines in panel (a). 
$2.2 \mathrm{nC}$ charge generated by using a $3 \mathrm{PW}, 350$ fs laser and $18 \mathrm{~m}$ long plasma channel with density $n=1.0 \times$ $10^{16} \mathrm{~cm}^{-3}$.

For high-energy physics applications, where the energy frontier exceeds $100 \mathrm{GeV}$, the synchrotron radiation of electron beams in the focusing fields of the wake can induce significant energy loss, energy spread, and emittance damping.

In order to reduce the energy spread electron beam with small spot size will be required. The energy spread can be estimated by calculating the power radiated by a single electron, $P=\frac{2}{3} \frac{e^{2}}{c} \gamma^{6}\left[(\dot{\vec{\beta}})^{2}-(\vec{\beta} \times \dot{\vec{\beta}})^{2}\right]$ [37]. For an electron executing betatron oscillations with a frequency $\omega_{p} / \sqrt{2 \gamma}$, the ratio of energy loss due to synchrotron radiation to the energy gain in the accelerating field is therefore

$$
\begin{aligned}
\frac{\epsilon_{r}}{\epsilon_{\mathrm{LW}}} \equiv & \frac{\Delta \gamma}{\gamma}=\frac{1}{6} \frac{1}{\epsilon_{\mathrm{LW}}} \frac{r_{e} \sigma_{r}^{2} \gamma^{2}}{\left(c / \omega_{p}\right)^{3}} \\
\approx & 1.2 \times 10^{-4} \frac{1}{\epsilon_{\mathrm{LW}}}\left(\sigma_{r}[\mu \mathrm{m}]\right)^{2}\left(E_{b}[100 \mathrm{GeV}]\right)^{2} \\
& \times\left(n_{p}\left[10^{16} \mathrm{~cm}^{-3}\right]\right)^{3 / 2},
\end{aligned}
$$

where $\epsilon_{r}$ is the normalized retarding field, $\epsilon_{\mathrm{LW}}$ is the normalized accelerating field, $r_{e}$ is the classical electron radius, and $\sigma_{r}$ is the spot size of the beam. For the $120 \mathrm{GeV}$ example given above where $\epsilon \sim 2, n_{p}=10^{16} \mathrm{~cm}^{-3}$, then in order for $\Delta \gamma / \gamma<10^{-2}$, the spot size $\sigma_{r}$ should be less than $10 \mu \mathrm{m}$. For such a spot size, a bunch length $\sim c / \omega_{p}$ and a charge of $2.2 \mathrm{nC}$, the ratio of the beam density to the plasma density is 17 .

The beam's spot size will decrease and hence the normalized emittance will also decrease because those electrons at the larger radii will radiate more. The normalized emittance scales as $\gamma \frac{\omega_{p}}{\sqrt{2 \gamma}} \sigma_{r}^{2}$ while $\frac{\omega_{p}}{\sqrt{2 \gamma}} \sigma_{r}^{2}$ is an adiabatic invariant. Therefore, $\frac{\Delta \epsilon_{N}}{\epsilon_{N}} \sim \frac{\Delta \gamma}{\gamma}$. A more detailed calculation of these effects for an ensemble of electrons was recently made by Michel et al. [38].

\section{CONCLUSION}

In conclusion, we have presented a phenomenological theory for LWFA in the nonlinear blowout regime. This theory includes the concepts of nonlinear multidimensional wake excitation, local pump depletion, dephasing, laser guiding, and beam loading. This theory provides a recipe for designing a LWFA for given laser and plasma parameters and estimates the number and the energy of the accelerated electrons whether self-injected or externally injected. These formulas apply for self-guided as well as externally guided pulses (e.g. by plasma channels). We have compared the scaling laws for this nonlinear regime with those derived for 1D linear, 1D nonlinear, and the recent $3 \mathrm{D}$ similarity theory. We have examined this regime via PIC simulations, and presented results of a $30 \mathrm{fs}$, $200 \mathrm{TW}$ laser interacting with a $0.75 \mathrm{~cm}$ long plasma with a density $1.5 \times 10^{18} \mathrm{~cm}^{-3}$. In this simulation an ultrashort (10 fs) monoenergetic bunch of self-injected electrons at $1.5 \mathrm{GeV}$ with $0.3 \mathrm{nC}$ of charge was observed. For future higher-energy accelerator applications (GeV and beyond), we propose to use externally injected electron beams to maintain high beam quality and to avoid dark current.

We close by commenting on how our work might be applied to the parameters of the recently published experimental results in which a $1 \mathrm{GeV}$ monoenergetic electron beam was observed. In this experiment a $40 \mathrm{TW} 40 \mathrm{fs}$ (12 TW $70 \mathrm{fs)} \mathrm{laser} \mathrm{was} \mathrm{propagated} \mathrm{in} \mathrm{a} \mathrm{shallow} \mathrm{plasma}$ channel of density $\sim 4.3 \times 10^{18} \mathrm{~cm}^{-3} \quad(\sim 3.2 \times$ $\left.10^{18} \mathrm{~cm}^{-3}\right)$. In the experiment, neither the laser spot size nor the pulse length was initially matched (initially $W_{0} \approx$ $25 \mu \mathrm{m})$. For example, for this density a $40 \mathrm{TW}$ laser should have a matched spot size $W_{0} \approx 2 \sqrt{2\left(\frac{P}{P_{c}}\right)^{1 / 3}} c / \omega_{p} \approx$ $10 \mu \mathrm{m}$ and a pulse length of $30 \mathrm{fs}$. Therefore, in this experiment the laser will evolve significantly, both longitudinally and transversely so that applying our scaling laws is not completely appropriate. Nevertheless, for these parameters, Eq. (6) predicts an output energy of $0.5 \mathrm{GeV}$, while the experimentally observed value was $1.0 \mathrm{GeV}$ which is within a factor of 2 . The interpretation of this experimental result is likely to be more similar to that in Tsung et al. [7], where a $16 \mathrm{TW}$ laser produced a somewhat monoenergetic beam at $0.7 \mathrm{GeV}$ (in Fig. 1 of this reference, the electron spectra were on a log scale). However, in the simulations of Tsung et al. the channel was narrower.

\section{ACKNOWLEDGMENTS}

The work is supported by DOE under Grants No. DEFC02-01ER41179, No. DE-FG02-03ER54721, No. DEFG03-92-ER4727, No. DE-FG03-NA0065, and No. DEFG52-06NA26195, by NSF under Grant No. Phy-0321345, and by FCT (Portugal). The simulations were performed on Dawson cluster, maintained locally by UCLA/ATS, and on the IBM SP at NERSC under mp113 and gc2. We acknowledge very useful conversations with Professor T. Katsouleas and Professor A. Pukhov.

\section{APPENDIX: PARAMETERS AND SETUP}

The simulation for the $200 \mathrm{TW}, 30 \mathrm{fs}$ laser pulse in a fully ionized $n=1.5 \times 10^{18} \mathrm{~cm}^{-3}$ plasma has a computational window of dimension $101.9 \times 127.3 \times 127.3 \mu \mathrm{m}^{3}$ which moves at the speed of light. The number of gridpoints is $4000 \times 256 \times 256=2.62 \times 10^{8}$. The resolution in the laser propagation direction $z$ is $k_{0} \Delta z=0.2$. We assume a preformed fully ionized plasma with uniform density profile. The resolution in the transverse direction is $k_{p} \Delta x=k_{p} \Delta y=0.116$. We use 2 electrons per cell and a smooth neutralizing immobile ion background (the total 
number of particles is roughly 500 million). A diffraction limited, circularly polarized pulse is focused at the plasma entrance with a spot size $w_{0}=19.5 \mu \mathrm{m}$ and the electric field has a symmetric temporal profile of $10 \tau^{3}-15 \tau^{4}+$ $6 \tau^{5}$, where $\tau=\sqrt{2}\left(t-t_{0}\right) / \tau_{\text {FWHM }}$. The laser is Gaussian in the transverse direction. The total axial length of the plasma is close to $0.75 \mathrm{~cm}$, or 3000000 simulation time steps.

The simulation for the $2 \mathrm{PW}, 100 \mathrm{fs}, w_{0}=40 \mu \mathrm{m}$ laser in plasma density $n=4 \times 10^{17}$ has a computational window of dimension $203.8 \times 127.3 \times 127.3 \mu \mathrm{m}^{3}$ which corresponds to $8000 \times 256 \times 256=5.24 \times 10^{8}$ gridpoints with identical resolution as in the aforementioned 200 TW run. Roughly 1 billion particles were used and ran 16000 simulation time steps. The rise time of the laser was about 2.5 times shorter than the fall time.

[1] T. Tajima and J. M. Dawson, Phys. Rev. Lett. 43, 267 (1979).

[2] P. Chen, J. M. Dawson, Robert W. Huff, and T. Katsouleas, Phys. Rev. Lett. 54, 693 (1985).

[3] Guo-Zheng Sun, Edward Ott, Y.C. Lee, and Pervez Guzdar, Phys. Fluids 30, 526 (1987).

[4] J. B. Rosenzweig, B. Breizman, T. Katsouleas, and J. J. Su, Phys. Rev. A 44, R6189 (1991).

[5] W. B. Mori, T. Katsouleas, C. B. Darrow, C. E. Clayton, C. Joshi, J. M. Dawson, C. B. Decker, K. Marsh, and S.C. Wilks, Proceedings of the Particle Accelerator Conference. San Francisco, California, 1991.

[6] A. Pukhov and J. Meyer-ter-vehn, Appl. Phys. B 74, 355 (2002).

[7] F. S. Tsung, Ritesh Narang, W. B. Mori, C. Joshi, R. A. Fonseca, and L. O. Silva, Phys. Rev. Lett., 93, 185002 (2004).

[8] M. Hogan et al., Phys. Rev. Lett. 95, 054802 (2005).

[9] I. Blumenfeld et al., Nature (London) 445, 741 (2007).

[10] S.P.D. Mangles et al., Nature (London) 431, 535 (2004).

[11] C. G. R. Geddes et al., Nature (London) 431, 538 (2004).

[12] J. Faure et al., Nature (London) 431, 541 (2004).

[13] S. P. D. Mangles et al., Phys. Rev. Lett. 96, 215001 (2006).

[14] C.-T. Hsieh, C.-M. Huang, C.-L. Chang, Y.-C. Ho, Y.-S. Chen, J.-Y. Lin, J. Wang, and S.-Y. Chen, Phys. Rev. Lett. 96, 095001 (2006).

[15] W. P. Leemans et al., Nature Phys. 2, 696 (2006).
[16] F. S. Tsung, W. Lu, M. Tzoufras, W. B. Mori, C. Joshi, J. M. Vieira, L. O. Silva, and R. A. Fonseca, Phys. Plasmas 13, 056708 (2006).

[17] N. H. Matlis et al., Nature Phys. 2, 749 (2006).

[18] T. Katsouleas, S. Wilks, P. Chen, J. M. Dawson, and J. J. Su, Part. Accel. 22, 81 (1987).

[19] P. Mora and T. M. Antonsen, Jr., Phys. Plasmas 4, 217 (1997).

[20] I. Kostyukov, A. Pukhov, and S. Kiselev, Phys. Plasmas 11, 5256 (2004).

[21] W. Lu, C. Huang, M. Zhou, W. B. Mori, and T. Katsouleas, Phys. Rev. Lett. 96, 165002 (2006).

[22] W. Lu, C. Huang, M. Zhou, M. Tzoufras, F. S. Tsung, W. B. Mori, and T. Katsouleas, Phys. Plasmas 13, 056709 (2006).

[23] V. Malka, J. Faure, Y. Glinnec, and A. F. Lifschitz, Plasma Phys. Controlled Fusion 47, B481 (2005).

[24] S. Gordienko and A. Pukhov, Phys. Plasmas 12, 043109 (2005).

[25] R. A. Fonseca et al., in ICCS 2002, LNCS 2331, edited by P. M. A. Sloot et al., 2002, pp. 342-351.

[26] E. Esarey, P. Sprangle, J. Krall, and A. Ting, IEEE Trans. Plasma Sci. 24, 252 (1996), and references therein.

[27] W. B. Mori, IEEE J. Quantum Electron. 33, 1942 (1997), and references therein.

[28] C. D. Decker, W. B. Mori, K.-C. Tzeng, and T. Katsouleas, Phys. Plasmas 3, 2047 (1996).

[29] L. M. Gorbunov, S. Yu. Kalmykov, and P. Mora, Phys. Plasmas 12, 033101 (2005).

[30] S. Yu. Kalmykov, L. M. Gorbunov, P. Mora, and G. Shvets, Phys. Plasmas 13, 113102 (2006).

[31] C. D. Decker and W. B. Mori, Phys. Rev. Lett. 72, 490 (1994); Phys. Rev. E 51, 1364 (1995).

[32] D. F. Gordon, B. Hafizi, R. F. Hubbard, J. R. Peano, P. Sprangle, and A. Ting, Phys. Rev. Lett. 90, 215001 (2003).

[33] F.S. Tsung, C. Ren, L. O. Silva, W. B. Mori, and T. Katsouleas, PANS V.99, No. 1, 2002, p. 29-32.

[34] C. Ren, B. J. Duda, R. G. Hemker, W. B. Mori, T. Katsouleas, T. M. Antonsen, Jr., and P. Mora, Phys. Rev. E 63, 026411 (2001).

[35] J. Faure et al., Phys. Rev. Lett. 95, 205003 (2005).

[36] J. Faure, C. Rechatin, A. Norlin, A. Lifschitz, Y. Glinnec, and V. Malka, Nature (London) 444, 737 (2006).

[37] J.D. Jackson, Classical Electrodynamics, 3rd Edition (John Wiley and Sons, Inc., New York, 1998).

[38] P. Michel, C. B. Schroeder, B. A. Shadwick, E. Esarey, and W. P. Leemans, Phys. Rev. E 74, 026501 (2006).

[39] S. Mallat, A Wavelet Tour of Signal Processing (Academic Press, London, 1998), pp. 104-113. 